7 Epler GR, Kelly EM. Systematic review of postradiotherapy bronchiolitis obliterans organizing pneumonia in women with breast cancer. Oncologist 2014; 19: 1216-1226.

8 Katayama N, Sato S, Katsui K, et al. Analysis of factors associated with radiation-induced bronchiolitis obliterans organizing pneumonia syndrome after breast-conserving therapy. Int J Radiat Oncol Biol Phys 2009; 73: 1049-1054.

9 Bhatt JM, Bush A, van Gerven M, et al. A statement on the multidisciplinary respiratory management of ataxia telangiectasia. Eur Respir Rev 2015; 24: 565-581.

10 Bhatt JM, Bush A, van Gerven M, et al. Ataxia telangiectasia: why should the ERS care? Eur Respir J 2015; 46: 1557-1560.

11 Schroeder SA, Swift M, Sandoval C, et al. Interstitial lung disease in patients with ataxia-telangiectasia. Pediatr Pulmonol 2005; 39: 537-543.

12 Zhang L, Yang M, Bi N, et al. ATM polymorphisms are associated with risk of radiation-induced pneumonitis. Int J Radiat Oncol Biol Phys 2010; 77: 1360-1368.

13 Ostendorf BN, Terwey TH, Hemmati PG, et al. Severe radiotoxicity in an allogeneic transplant recipient with a heterozygous ATM mutation. Eur J Haematol 2015; 95: 90-92.

14 Renwick A, Thompson D, Seal S, et al. ATM mutations that cause ataxia-telangiectasia are breast cancer susceptibility alleles. Nat Genet 2006; 38: 873-875.

\title{
Regimens for nontuberculous mycobacterial lung disease lack early bactericidal activity
}

\author{
To the Editor:
}

Over recent decades, nontuberculous mycobacteria (NTM) have been increasingly recognised as causative agents of pulmonary infections in humans [1]. Mycobacterium avium complex (MAC) bacteria are the most common NTM species causing pulmonary disease in humans [1]. Pulmonary disease caused by NTM (NTM-PD) usually presents as either nodular-bronchiectatic or fibrocavitary disease. NTM-PD requires complex multidrug antibiotic treatment to be continued for 12 months after culture conversion, typically for 18 months in total [1]. Despite this intensive treatment, good clinical and microbiological outcomes can only be attained in $60 \%$ of patients with MAC pulmonary disease [2]. Outcomes in nodular-bronchiectatic MAC pulmonary disease are better, with prolonged culture conversion attainable in up to $85 \%$ of patients [3].

The long treatment regimen, and the bias introduced by morbidity and mortality related to the patients' comorbid conditions and comedications, complicate the use of long-term outcome measures in clinical trials. Hence, there is a need for a robust marker that can be measured early in treatment and that predicts the long-term outcome of treatment. One such marker could be the early bactericidal activity (EBA). EBA studies are based on the principle that the effect of treatment in the first days or weeks on the bacillary load in clinical samples predicts the long-term result of treatment. The treatment of tuberculosis has been optimised using this principle [4].

If they prove to be good predictors of long-term outcomes, EBA studies could greatly decrease the time required to assess the effect of treatment. In this pilot study, we aimed to measure EBA of current treatment regimens for NTM-PD.

Three consecutively admitted patients were followed with weekly spot sputum cultures, which is part of the routine clinical care in the first phase of treatment. Patient 1 was a 68-year-old female with an unremarkable clinical history diagnosed with nodular-bronchiectatic Mycobacterium intracellulare pulmonary disease. Patient 2 was a 74-year-old female with a history of asthma and diagnosed with nodular-bronchiectatic Mycobacterium chelonae pulmonary disease. Patient 3 was a 65 -year-old female with a history of chronic obstructive pulmonary disease, now diagnosed with severe fibrocavitary $M$. avium pulmonary disease.

Alongside routine cultures, we performed quantitative cultures to assess the mycobacterial load in the sputum samples. The patients were followed the first month to assess the early bactericidal activity. In total, five sputum samples were collected from every patient. The first sample was collected before the start of treatment; thereafter, we collected a spot sputum sample each week for 1 month. The first (baseline) sputum was collected while patients were admitted; thereafter, patients collected sputum at home once a 
week. Pre-treatment isolates were identified by the Inno-LiPA line probe assay (Innogenetics, Ghent, Belgium) and subjected to antimicrobial susceptibility testing by broth microdilution [5].

The sputum was digested using $0.1 \%$ dithiothreitol (Sputasol; Oxoid, Landsmeer, the Netherlands). Dilutions ranging from $10^{0}$ to $10^{-4}$ were made using sterile $0.85 \%$ saline. From these, $100 \mu \mathrm{L}$ was inoculated in duplicate onto Middlebrook 7H11 agar plates (BD Bioscience, Erembodegem, Belgium), enriched with oleic acid, bovine albumin, dextrose and catalase supplement (OADC; BD Bioscience), and made selective by adding polymyxin $\mathrm{B}$, amphotericin B, nalidixic acid, trimethoprim and azlocillin antibiotic supplement (PANTA; BD Bioscience). The plates were incubated at $37^{\circ} \mathrm{C}$ in ambient air, examined after 2 days for contamination and read weekly for 4 weeks. Colonies were counted when they were easily visible and before they coalesced. Ziehl-Neelsen staining was performed to distinguish between acid-fast bacilli and contaminants. After counting, the plates were re-incubated and recounted after 1 week to ensure accurate counts. The counts from the two duplicate measurements were used to calculate the mean number of colony-forming units (cfu) per millilitre of sputum. The result was expressed as the $\log _{10} \mathrm{cfu} \cdot \mathrm{mL}^{-1}$ of the undiluted sputum.

Patient 1 started treatment with rifampicin, ethambutol and azithromycin; patient 2 with inhaled tobramycin and oral ciprofloxacin; and patient 3 with clofazimine, amikacin, rifampicin, ethambutol and azithromycin. All patients continued treatment for the first month; none changed regimens because of intolerance.

Results of the cfu counts over time are shown in figure 1. cfu counts showed a minor decrease of $0.5 \log _{10} \mathrm{cfu} \cdot \mathrm{mL}^{-1}$ over 4 weeks (patients 1 and 3 ) or remained stable during the month of treatment (patient 2). All pre-treatment MAC isolates were susceptible to clarithromycin and had minimum inhibitory concentrations (MICs) $<32 \mathrm{mg} \cdot \mathrm{L}^{-1}$ for amikacin. The $M$. chelonae isolate was susceptible to tobramycin and intermediately susceptible to ciprofloxacin (MIC $2 \mathrm{mg} \cdot \mathrm{L}^{-1}$ ).

Our small study suggests that currently recommended treatment regimens for NTM-PD have very limited early bactericidal activity and perhaps only exert a slow sterilising capacity. Introduction of a drug with EBA may accelerate culture conversion and, given that treatment is to be continued for 12 months after culture conversion [1], shorten the treatment of NTM-PD. This may be more relevant for the fibrocavitary NTM-PD, with its high bacterial burden [1], than for nodular-bronchiectatic NTM-PD. No new drugs with likely EBA in NTM-PD are currently in the pipeline, but aminoglycosides might prove useful. Streptomycin accelerated sputum culture conversion, suggestive of some EBA, but did not impact long-term treatment outcomes [6]. We saw no significant EBA of amikacin in patient 3. In tuberculosis, amikacin shows very little EBA (0.041-0.045 $\left.\log _{10} \mathrm{cfu} \cdot \mathrm{day}^{-1}\right)$ compared to rifampicin $\left(0.20-0.63 \log _{10} \mathrm{cfu} \cdot \mathrm{day}^{-1}\right)$, isoniazid $\left(0.37-0.77 \log _{10} \mathrm{cfu} \cdot \mathrm{day}^{-1}\right)$ and even ethambutol $\left(0.05 \log _{10} \mathrm{cfu} \cdot \mathrm{day}^{-1}\right)$. EBA of these single drugs in tuberculosis is higher than what we measured for the multidrug therapy for NTM-PD [6]. This probably reflects the intrinsic resistance of NTM to antimicrobial drugs. Higher EBA in NTM-PD might be achieved if the concentration of these drugs at the site of infection could be increased. To achieve this, trials of inhaled liposomal amikacin are currently in progress [7].

During 6 months of follow-up, patient 1 had to switch from rifampicin to clofazimine due to gastrointestinal side-effects, reported symptomatic improvement and converted to negative cultures after 4 months of treatment. Patient 2 reported amelioration of symptoms, but showed slight progression of radiological abnormalities and remained culture positive after 5 months; she has since switched to a tobramycin-azithromycin regimen. Patient 3 successfully completed 3 months of amikacin and clofazimine,

FIGURE 1 Mycobacterial load in $\log _{10}$ colony-forming units (cfu) per millilitre of sputum over 4 weeks of treatment in three patients.

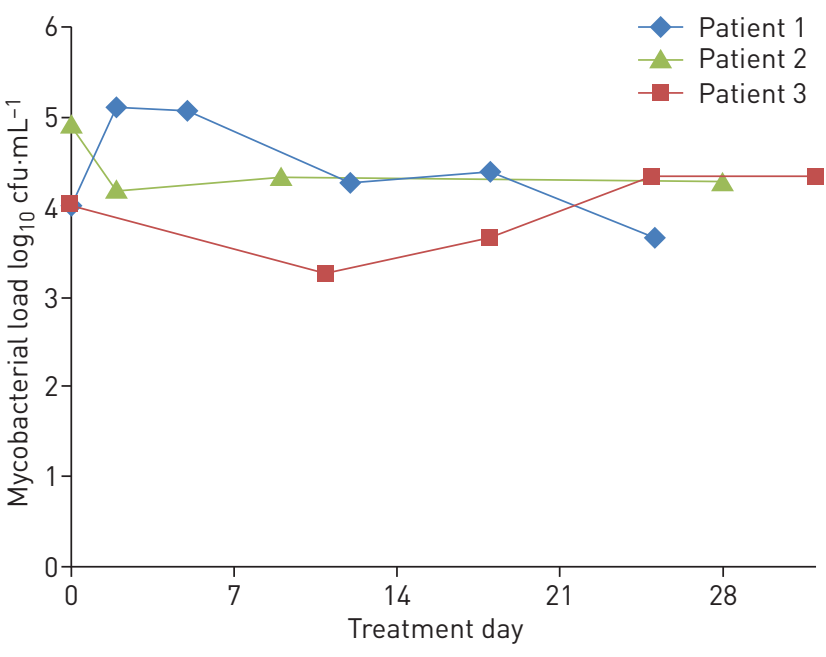


has continued on rifampicin, ethambutol and azithromycin, and has reported clinical improvement. She achieved a negative culture after 3 months of treatment; those taken after 6 months were pending at the time of writing. The two patients who showed the slight reduction in $\mathrm{cfu} \cdot \mathrm{mL}^{-1}$ sputum were the two who converted to negative cultures after 4 and 5 months of treatment, while patient 2 did not show any reduction and did not convert. This hints at a possible predictive value of the EBA for the chance to attain culture conversion during the first 6 months and this should be subject of further study.

There are several limitations to this small pilot study. First, we used only spot sputum samples, which may result in more variable cfu counts in comparison with overnight sputum samples [8]. Second, because no previous data on EBA in NTM-PD existed, we included patients with NTM-PD regardless of manifestation or causative species.

Based on our sample of three patients, we did not find evidence for EBA of currently recommended treatment regimens for NTM-PD. Slight decreases in mycobacterial load during the first month might predict whether culture conversion occurs in the first 6 months of treatment. Treatment of NTM-PD may be improved by adding or replacing one of the current drugs with a drug that does add EBA, which could lead to faster sputum conversion and, therefore, to shorter treatment.

- $@$ ERSpublications

Treatment regimens for NTM lung disease lack early bactericidal activity and must be improved http://ow.ly/UyFdu

Michiel H.C. Slaats ${ }^{1}$, Wouter Hoefsloot ${ }^{2}$, Cecile Magis-Escurra ${ }^{2}$, Martin J. Boeree ${ }^{2}$, Melanie Wattenberg ${ }^{1}$, Saskia Kuipers ${ }^{1}$ and Jakko van Ingen ${ }^{1}$

${ }^{1}$ Dept of Medical Microbiology, Radboud University Medical Center, Nijmegen, The Netherlands. ${ }^{2}$ Dept of Respiratory Diseases, Radboud University Medical Center, Nijmegen, The Netherlands.

Correspondence: Jakko van Ingen, Dept of Medical Microbiology (777), PO Box 9101, 6500HB Nijmegen, The Netherlands. E-mail: Jakko.vaningen@radboudumc.nl

Received: June 122015 | Accepted after revision: Nov 022015 | First published online: Dec 032015

Conflict of interest: None declared.

\section{References}

1 Griffith DE, Aksamit T, Brown-Elliott BA, et al. An official ATS/IDSA statement: diagnosis, treatment, and prevention of nontuberculous mycobacterial diseases. Am J Respir Crit Care Med 2007; 175: 367-416.

2 van Ingen J, Ferro BE, Hoefsloot W, et al. Treatment of nontuberculous mycobacterial lung disease in HIV-negative patients - the evidence. Expert Rev Anti Infect Ther 2013; 11: 1065-1077.

3 Wallace R, Brown-Elliott B, McNulty S, et al. Macrolide/azalide therapy for nodular/bronchiectatic Mycobacterium avium complex lung disease. Chest 2014; 146: 276-282.

4 Donald PR, Diacon AH. The early bactericidal activity of anti-tuberculosis drugs: a literature review. Tuberculosis 2008; 88: Suppl. 1, S75-S83.

5 Clinical Laboratory Standards Institute. Susceptibility Testing of Mycobacteria, Nocardiae, and Other Aerobic Actinomycetes - Approved Standard, Second Edition. CLSI document M24-A2. Wayne, Clinical Standards Institute, 2011.

6 Kobashi Y, Matsushima T, Oka M. A double-blind randomized study of aminoglycoside infusion with combined therapy for pulmonary Mycobacterium avium complex disease. Respir Med 2007; 101: 130-138.

7 Olivier KN, Gupta R, Daley CL, et al. A randomized, double-blind, placebo-controlled study of liposomal amikacin for inhalation (Arikace ${ }^{\circledast}$ ) in patients with recalcitrant nontuberculous mycobacterial lung disease. Am J Respir Crit Care Med 2014; 189: A4126.

8 Hafner R, Cohn J, Wright D, et al. Early bactericidal activity of isoniazid in pulmonary tuberculosis: optimizing of methodology. Am J Respir Crit Care Med 1997; 156: 918-923. 